\title{
Buying Behaviour and Preferences of Consumers with Reference to Fmcg Goods in North Coastal Andhra Pradesh
}

\author{
ShaikShamshuddin, T. Venkateswarulu, UV adinarayana, I. Bangaruraju, SG Ramarao
}

\begin{abstract}
The world has become a village and many MNCs have entered Indian operations as it is an attractive destiny for the burgeoning middle class with its increase in disposable income. This competition opened the new doors and ways to face the challenges encountering from FMCG Markets. There are many studies that analyse the consumer brand loyalty towards FMCG products, consumer buying behaviour and brand loyalty of the fast moving consumer goods in the rural markets, the contribution of FMCG goodsin economy of country, factors influencing demand of FMCG goodsamong people. Also there are studies that analyses the purchase behaviour of the male and female separately towards the FMCG goods. But no study has been done on buying behaviour of consumers in FMCG sector in the North Coastal Districts of Andhra Pradesh. Hence this Research Paper concentrated on North Coastal Andhra Pradesh Consumers and their buying behavior. The study of consumer preferences towards fast moving consumer goods relates to the buying preferences of FMCG products based on product attributes, price, quality etc. Before finalizing on the questionnaire a pilot study was carried out. This was to primarily check on the accuracy of the questions used in the survey. Field survey was carried out. This questionnaire was administered to 150 respondents. This Article is done with help of both sources like primary and secondary data. Articleconnected with the three districts of North Coastal Andhra Pradesh and consumers were selected both from rural and urban locations in each district.
\end{abstract}

Key words: Consumer, Decision, Industry, Selection, Process, Behavior

\section{INTRODUCTION}

Consumers constantly make decisions regarding the choice, purchase, and use of products and services. The decisions are of great importance not only for the consumers themselves but also for marketers and policy makers. These decisions are often difficult. Consumers are often faced with a large number of alternatives, which are constantly increasing due to new technologies and competitive pressures. Products that have quick turnover and relatively low cost are termed as Fast Moving Consumer Goods (FMCG). FMCG has a strong MNC presence and is characterized by a well-established distribution network,

Revised Manuscript Received on April 15, 2020.

* Correspondence Author

Dr.ShaikShamshuddin, Assistant Professor, GITAM Institute of Management, GITAM Deemed to be University.

Dr. T. Venkateswarulu, Assistant Professor, GITAM Institute of Management, GITAM Deemed to be University.

Dr. UV adinarayana, Assistant Professor, GITAM Institute of Management, GITAM Deemed to be University.

Dr. I. Bangaruraju, Associate Professor, GITAM Institute of Management, GITAM Deemed to be University

Dr.SG Ramarao, Assistant Professor, GITAM Institute of Management, GITAM Deemed to be University.

(C) The Authors. Published by Blue Eyes Intelligence Engineering and Sciences Publication (BEIESP). This is an open access article under the CC BY-NC-ND license (http://creativecommons.org/licenses/by-nc-nd/4.0/)
The products of fast moving consumer goods are generally named as that of consumer packaged goods. The products in intense competition between the organized and unorganized segments and low operational cost. this category comprise of all the consumables (except the pulses / groceries) that people purchase at standard intervals.

The consumers in rural households preferred unbranded products. Consumers in rural India are now buying branded, specialized utensil cleaners and shifted from the typical soil or ash in the past. The penetration of the branded products has also increased significantly for the items such as detergent cakes, tooth paste, hair oils and more. Since the price is low FMCG products have recorded considerable growth in the rural areas.

\section{A. Problem Identification:}

The industry of fast moving consumer goods offers a good number of products at customer and consumer convinience. Also there is huge competition among the manufacturers of FMCG products. This results in the increased investment in the FMCG sector. Particularly India is ranked as the fourth major sector and the FMCG sector acts a significant role itself. So it is very important to study the consumer behaviour of the. The problem identified in this study is that there is no study that exclusively deals with the buying preferences of customers towards the FMCG goods in the northern part of AP state. Hence this study would analyze the consumer preference towards select FMCG in the North Coastal Andhra Pradesh in India and help the marketers of Andhra Pradesh in formulating marketing strategies that would serve their consumers in a better way.

\section{B. Review of Literature:}

The political and socio economic factors brought about these alterations in the life styles of the people in the countryside, who have started patronizing branded products of FMCG. In the state of Andhra Pradesh the speedy development of the degree \& engineering colleges and the rural polytechnic colleges are giving easy access to educationto the rural people. The policies of Government to endorse the education in the rural areas enhanced their awareness of the brand because of the existence of at least one higher education pursuing person in their family or in their neighbouring family (Amarnath\&Vijayudu, 2009).This highly contributes to awareness towards the branded products of FMCG.According to Huhmann and Sara (2004) brand ambassadors play as significant role in developing an impact on the brand preferences of rural India. .Promotion of brands requires special measures in the rural market. Branded products rarely fight for the share in the market; they have to be promoted in the right place.

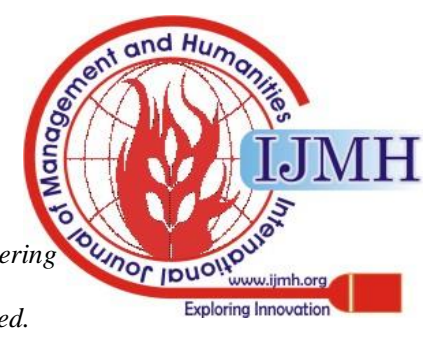


Brands such as Marie biscuits, clinic shampoo, close up are doing well because of proper distribution. Some of the brands are doing well without much promotion and advertisement. The main sources of information for consumer in the rural areas are newspapers, TV, cinema, radios, retailers and more.

\section{RESEARCH METHODOLOGY}

There are many studies that analyse the consumer brand loyalty towards FMCG products, consumer buying behaviour and brand loyalty of the fast moving consumer goods in the rural markets, the contribution of fast moving consumer goods in the economy of India, factors influencing the demand of the fast moving consumer goods among the people. Also there are studies that analyses the purchase behaviour of the male and female separately towards the fast moving consumer goods. But no study has been done on buying behaviour of consumers in FMCG sector in the North Coastal Districts of Andhra Pradesh.

\section{Objectives of the Study:}

- To assess the factors affecting buying decision of selecting FMCG products in general;

- $\quad$ To examine the type of media through which consumers are aware of FMCG product brands;

\begin{tabular}{|c|c|c|c|}
\hline S.NO & DISTRICT & CALCULATION SAMPLE & $\begin{array}{l}\text { RESPONDENTS } \\
\text { SELECTED }\end{array}$ \\
\hline 1 & Visakhapatnam & $900 \times 40,53,463 / 90,01,051$ & 405 \\
\hline 2 & Vizianagaram & $900 \times 23,44,474 / 90,01,051$ & 234 \\
\hline 3 & Srikakulam & $900 \times 27,03,114 / 90,01,051$ & 271 \\
\hline & Total & $91,01,051$ & 900 \\
\hline
\end{tabular}

iv. Limitations of Study:

- Only few FMCG products are selected for the Research.

- The Research has been confined to consumer preferences of selected FMCG goods.

- The study has been restricted to North Coastal Andhra Pradesh region only.

- The outcomes of this study are confined to North Coastal Andhra Pradesh.

- $\quad$ The sample size is limited to only 837 respondents from the three districts of North Coastal Andhra Pradesh.
- $\quad$ To identify the influence of product specific attributes of select FMCG products;

- To establish the relation of store attributes like brand and

- To understand the post purchase behaviour of customers of selected FMCG products;

i. Sample size Distribution:

This Research based on both primary data and secondary data. The study relates to the three districts of North Coastal Andhra Pradesh and consumers were selected both from rural and urban locations in each district. The primary data is collected from the consumers living in all the three districts of north coastal Andhra Pradesh

nh. $=$ SS $x$ SP / TP

NH=Size of selected district

SS=Sample size $\quad$ SP = Sub population size

$\mathbf{T P}=$ Total population size

ii. Statistical tools for the study:

- Chi-Square

- Factor Analysis

- F-Test and Percentage

iii. Sample size of Each District

\section{v. Analysis of the Data:}

Analysis was made in descriptive form and in percentages for all the questions in the questionnaire. The data analysis is classified into three major divisions which are as below.

Personal Profile of the respondents.

General Marketing information.

Brand Preferences of select FMCG Products.

\section{vi. Age wise classification of the respondents:}

\begin{tabular}{|c|c|c|}
\hline Age & Respondents & Percent \\
\hline Below 18 years & 51 & 6.1 \\
\hline 18-24 years & 114 & 13.6 \\
\hline 25-34 years & 179 & 21.4 \\
\hline
\end{tabular}




\begin{tabular}{|c|c|c|}
\hline 35-40 years & 250 & 29.9 \\
\hline 41-60 years & 222 & 26.5 \\
\hline Above 60 years & 21 & 2.5 \\
\hline & $\mathbf{8 3 7}$ & $\mathbf{1 0 0}$ \\
\hline
\end{tabular}

Source: Primary data

Nearly 30\% consumers are between age group of 35-40. 26.5 percent between 41-60 years, 21.4 percent in 25-34 years, 13.6 percent in 18-24 years, and 6.1 percent below 18 years and very few, that is, 2.5 percent of consumers are in above 60 years age group. It can be concluded that 35-40 years age group are more interested in buying FMCG products.

vii. Marital Status Wise Classification of the Respondents:

\begin{tabular}{|l|l|l|}
\hline Marital Status & Respondents & Percent \\
\hline Single/Unmarried & 267 & 31.9 \\
\hline Married & 570 & 68.1 \\
\hline Total & $\mathbf{8 3 7}$ & $\mathbf{1 0 0}$ \\
\hline
\end{tabular}

Source: Primary Data

It reveals that out of most of consumers are married.

viii. Education wise classification of the Respondents:

\begin{tabular}{|l|l|l|}
\hline Education & Respondents & Percent \\
\hline High school & 171 & 20.4 \\
\hline Graduate/ Diploma & 323 & 38.6 \\
\hline Post graduate & 260 & 31.1 \\
\hline Others & 83 & 9.9 \\
\hline Total & $\mathbf{8 3 7}$ & $\mathbf{1 0 0 . 0}$ \\
\hline
\end{tabular}

It is understood that most of the respondents are educated.

ix. Respondents by income:

\begin{tabular}{|l|l|l|}
\hline (in Rupees) & Consumers & Percent \\
\hline Below 10000 & 129 & 15.4 \\
\hline $10001-25000$ & 144 & 17.2 \\
\hline $25001-50000$ & 217 & 25.9 \\
\hline Above 50000 & 347 & 41.5 \\
\hline Total & $\mathbf{8 3 7}$ & $\mathbf{1 0 0}$ \\
\hline
\end{tabular}

Source: Primary data

Majority of the respondents are in above Rs.50000 income group.

x. Occupational classification of the Respondents: 
Buying Behaviour and Preferences of Consumers with Reference to Fmcg Goods in North Coastal Andhra Pradesh

\begin{tabular}{|c|c|c|}
\hline Occupation & Respondents & Percent \\
\hline Agriculture & 193 & 23.1 \\
\hline Business & 151 & 18.0 \\
\hline Government employee & 170 & 20.3 \\
\hline Private employee & 183 & 21.9 \\
\hline Student & 78 & 9.3 \\
\hline Home maker & 50 & 6.0 \\
\hline Others & 12 & 1.4 \\
\hline Total & $\mathbf{8 3 7}$ & $\mathbf{1 0 0 . 0}$ \\
\hline
\end{tabular}

Source: Primary Data

It is understood that majority of them are dependent on agriculture.

xi. Family classification:

\begin{tabular}{|c|c|c|}
\hline Classification & Consumers & Percent \\
\hline Nuclear & 694 & 82.9 \\
\hline Joint & 143 & 17.1 \\
\hline Total & $\mathbf{8 3 7}$ & $\mathbf{1 0 0}$ \\
\hline
\end{tabular}

Majority are from nuclear families.

xii. Media through which respondents got awareness about FMCG products:

\begin{tabular}{|l|l|l|}
\hline Media Source & Respondents & Percent \\
\hline Television & 526 & 62.8 \\
\hline Radio & 17 & 2.0 \\
\hline News paper & 52 & 6.2 \\
\hline Pamphlet & 26 & 3.1 \\
\hline Magazine & 26 & 3.1 \\
\hline Friends/Neighbours & 69 & 8.2 \\
\hline Shop Display & 91 & 10.9 \\
\hline Any other & 30 & 3.6 \\
\hline Total & $\mathbf{8 3 7}$ & $\mathbf{1 0 0}$ \\
\hline
\end{tabular}

It is stated that majority of the consumers came to know about FMCG products through television.

xiii. Preferred Place opted to purchase FMCG products:

\begin{tabular}{|l|l|l|}
\hline Purchase Place & Respondents & Percent \\
\hline Nearest Shop & 348 & 41.6 \\
\hline Shop in Town & 160 & 19.1 \\
\hline Big Retail Store & 297 & 35.5 \\
\hline Any other & 32 & 3.8 \\
\hline & $\mathbf{8 3 7}$ & $\mathbf{1 0 0}$ \\
\hline
\end{tabular}


It is understood that most of the respondents are preferring nearest shop to buy FMCG products as they are consumed on a regular basis and some preferring big retail store to buy these FMCG products along with other products. xiv. Factors influencing buying decision of FMCG product:

\begin{tabular}{|l|l|l|l|l|l|l|l|l|l|l|l|l|}
\hline Factors & $\mathbf{1}$ & $\mathbf{2}$ & $\mathbf{3}$ & $\mathbf{4}$ & $\mathbf{5}$ & $\mathbf{6}$ & $\mathbf{7}$ & $\mathbf{8}$ & $\mathbf{9}$ & $\mathbf{T o t a l}$ & $\begin{array}{l}\text { Total } \\
\text { Score }\end{array}$ & $\begin{array}{l}\text { Mean } \\
\text { Score }\end{array}$ \\
\hline Location & 184 & 420 & 11 & 8 & 88 & 8 & 25 & 67 & 26 & $\mathbf{8 3 7}$ & $\mathbf{5 8 4 8}$ & $\mathbf{6 . 9 9}$ \\
\hline $\begin{array}{l}\text { Product and Brand } \\
\text { Range }\end{array}$ & 485 & 181 & 9 & 16 & 24 & 84 & 8 & 7 & 23 & $\mathbf{8 3 7}$ & $\mathbf{6 4 8 9}$ & $\mathbf{7 . 7 5}$ \\
\hline Credit Facility & 33 & 93 & 407 & 186 & 8 & 6 & 86 & 10 & 8 & $\mathbf{8 3 7}$ & $\mathbf{5 3 5 6}$ & $\mathbf{6 . 4 0}$ \\
\hline $\begin{array}{l}\text { Friendly nature of } \\
\text { Shopkeeper }\end{array}$ & 7 & 27 & 274 & 8 & 76 & 424 & 7 & 5 & 9 & $\mathbf{8 3 7}$ & $\mathbf{4 3 6 1}$ & $\mathbf{5 . 2 1}$ \\
\hline Price and quality & 13 & 88 & 56 & 420 & 8 & 204 & 16 & 17 & 15 & $\mathbf{8 3 7}$ & $\mathbf{4 6 8 6}$ & $\mathbf{5 . 6 0}$ \\
\hline $\begin{array}{l}\text { Shop of known } \\
\text { person }\end{array}$ & 78 & 4 & 56 & 5 & 433 & 8 & 238 & 10 & 5 & $\mathbf{8 3 7}$ & $\mathbf{4 0 9 2}$ & $\mathbf{4 . 8 9}$ \\
\hline Home delivery & 7 & 5 & 8 & 90 & 7 & 13 & 30 & 672 & 5 & $\mathbf{8 3 7}$ & $\mathbf{2 2 2 5}$ & $\mathbf{2 . 6 6}$ \\
\hline Discount & 21 & 9 & 8 & 97 & 192 & 10 & 19 & 27 & 454 & $\mathbf{8 3 7}$ & $\mathbf{2 4 6 4}$ & $\mathbf{2 . 9 4}$ \\
\hline Bundled schemes & 8 & 13 & 11 & 7 & 8 & 80 & 396 & 21 & 293 & $\mathbf{8 3 7}$ & $\mathbf{2 1 7 8}$ & $\mathbf{2 . 6 0}$ \\
\hline
\end{tabular}

Source: Primary Data

It can be concluded that product and brand range followed by location are considered as important factors affecting buying decision of FMCG products.

\begin{tabular}{|c|c|c|c|c|c|c|c|}
\hline Type of discount & 1 & 2 & 3 & 4 & Total & $\begin{array}{l}\text { Total } \\
\text { Score }\end{array}$ & $\begin{array}{l}\text { Mean } \\
\text { Score }\end{array}$ \\
\hline Price Discount & $\begin{array}{l}335 \\
(40.0)\end{array}$ & $\begin{array}{l}244 \\
(29.2)\end{array}$ & $\begin{array}{l}247 \\
(29.5)\end{array}$ & $\begin{array}{l}11 \\
(1.3)\end{array}$ & $\begin{array}{l}837 \\
(\mathbf{1 0 0 . 0})\end{array}$ & 2577 & 3.08 \\
\hline Schemes like buy 2 get 1 free & $\begin{array}{l}422 \\
(50.4)\end{array}$ & $\begin{array}{l}262 \\
(31.3)\end{array}$ & $\begin{array}{l}85 \\
(10.2)\end{array}$ & $\begin{array}{l}68 \\
(8.1)\end{array}$ & $\begin{array}{l}837 \\
(100.0)\end{array}$ & 2712 & 3.24 \\
\hline $\begin{array}{l}\text { Bundled offer with another } \\
\text { product }\end{array}$ & $\begin{array}{l}20 \\
(2.4)\end{array}$ & $\begin{array}{l}165 \\
(19.7)\end{array}$ & $\begin{array}{l}460 \\
(55.0)\end{array}$ & $\begin{array}{l}192 \\
(22.9)\end{array}$ & $\begin{array}{l}837 \\
(100.0)\end{array}$ & 1687 & 2.02 \\
\hline Free Gift & $\begin{array}{l}61 \\
(7.3)\end{array}$ & $\begin{array}{l}167 \\
(20.0)\end{array}$ & $\begin{array}{l}51 \\
(6.1)\end{array}$ & $\begin{array}{l}558 \\
(66.7)\end{array}$ & $\begin{array}{l}837 \\
(\mathbf{1 0 0 . 0 )}\end{array}$ & 1405 & 1.68 \\
\hline
\end{tabular}

Source: Primary Data

It can be concluded that buy 2 get 1 free is the most preferred promotional offer of the sample respondents.

xvi. Factors influencing buying decisions of FMCG xv. Promotional offer preferences of respondents (Rank Order):

\section{Products:}


Buying Behaviour and Preferences of Consumers with Reference to Fmcg Goods in North Coastal Andhra Pradesh

\begin{tabular}{|c|c|c|c|c|c|c|}
\hline Factors & Dis agree & $\begin{array}{l}\text { Strongly } \\
\text { Disagree }\end{array}$ & $\begin{array}{l}\text { Neither agree } \\
\text { nor disagree }\end{array}$ & Agree & $\begin{array}{l}\text { Strongly } \\
\text { agree }\end{array}$ & Total \\
\hline $\begin{array}{l}\text { Changing financial } \\
\text { position }\end{array}$ & $\begin{array}{c}148 \\
(17.7)\end{array}$ & $\begin{array}{c}225 \\
(26.9)\end{array}$ & $\begin{array}{c}227 \\
(27.1)\end{array}$ & $\begin{array}{c}165 \\
(19.7)\end{array}$ & $\begin{array}{c}72 \\
(8.6)\end{array}$ & $\begin{array}{c}837 \\
(\mathbf{1 0 0 . 0 )}\end{array}$ \\
\hline $\begin{array}{l}\text { Emerging beauty } \\
\text { consciousness }\end{array}$ & $\begin{array}{c}161 \\
(19.2)\end{array}$ & $\begin{array}{c}162 \\
(19.4)\end{array}$ & $\begin{array}{c}113 \\
(13.5)\end{array}$ & $\begin{array}{c}310 \\
(37.0)\end{array}$ & $\begin{array}{c}91 \\
(10.9)\end{array}$ & $\begin{array}{c}837 \\
(\mathbf{1 0 0 . 0 )}\end{array}$ \\
\hline To protect health & $\begin{array}{c}95 \\
(11.4)\end{array}$ & $\begin{array}{c}30 \\
(3.6)\end{array}$ & $\begin{array}{c}73 \\
(8.7)\end{array}$ & $\begin{array}{c}316 \\
(37.8)\end{array}$ & $\begin{array}{c}323 \\
(38.6)\end{array}$ & $\begin{array}{c}837 \\
(\mathbf{1 0 0 . 0 )}\end{array}$ \\
\hline To be fashionable & $\begin{array}{c}63 \\
(7.5)\end{array}$ & $\begin{array}{c}87 \\
(10.4)\end{array}$ & $\begin{array}{c}116 \\
(13.9)\end{array}$ & $\begin{array}{c}399 \\
(47.7)\end{array}$ & $\begin{array}{c}172 \\
(20.5)\end{array}$ & $\begin{array}{c}837 \\
(\mathbf{1 0 0 . 0 )}\end{array}$ \\
\hline $\begin{array}{l}\text { To live healthy and } \\
\text { hygienic life }\end{array}$ & $\begin{array}{c}84 \\
(10.0)\end{array}$ & $\begin{array}{c}24 \\
(2.9)\end{array}$ & $\begin{array}{c}73 \\
(8.7)\end{array}$ & $\begin{array}{c}267 \\
(31.9)\end{array}$ & $\begin{array}{c}389 \\
(46.5)\end{array}$ & $\begin{array}{c}837 \\
(\mathbf{1 0 0 . 0 )}\end{array}$ \\
\hline To withstand pollution & $\begin{array}{c}53 \\
(6.3)\end{array}$ & $\begin{array}{c}143 \\
(17.1)\end{array}$ & $\begin{array}{c}90 \\
(10.8)\end{array}$ & $\begin{array}{c}330 \\
(39.4)\end{array}$ & $\begin{array}{c}221 \\
(26.4)\end{array}$ & $\begin{array}{c}837 \\
(\mathbf{1 0 0 . 0 )}\end{array}$ \\
\hline $\begin{array}{l}\text { To change along with } \\
\text { changes in civilization }\end{array}$ & $\begin{array}{c}107 \\
(12.8)\end{array}$ & $\begin{array}{c}96 \\
(11.5)\end{array}$ & $\begin{array}{c}133 \\
(15.9)\end{array}$ & $\begin{array}{c}341 \\
(40.7)\end{array}$ & $\begin{array}{c}160 \\
(19.1)\end{array}$ & $\begin{array}{c}837 \\
(\mathbf{1 0 0 . 0 )}\end{array}$ \\
\hline $\begin{array}{l}\text { Sales and promotional } \\
\text { activities of firms }\end{array}$ & $\begin{array}{c}76 \\
(9.1)\end{array}$ & $\begin{array}{c}209 \\
(25.0)\end{array}$ & $\begin{array}{c}255 \\
(30.5)\end{array}$ & $\begin{array}{c}191 \\
(22.8)\end{array}$ & $\begin{array}{c}106 \\
(12.7)\end{array}$ & $\begin{array}{c}837 \\
(\mathbf{1 0 0 . 0 )}\end{array}$ \\
\hline $\begin{array}{l}\text { Low-unit price of FMCGs } \\
\text { products }\end{array}$ & $\begin{array}{c}88 \\
(10.5)\end{array}$ & $\begin{array}{c}186 \\
(22.2)\end{array}$ & $\begin{array}{c}238 \\
(28.4)\end{array}$ & $\begin{array}{c}183 \\
(21.9)\end{array}$ & $\begin{array}{c}142 \\
(17.0)\end{array}$ & $\begin{array}{c}837 \\
(100.0)\end{array}$ \\
\hline $\begin{array}{l}\text { Impressed by } \\
\text { advertisements }\end{array}$ & $\begin{array}{c}66 \\
(7.9)\end{array}$ & $\begin{array}{c}193 \\
(23.1)\end{array}$ & $\begin{array}{c}274 \\
(32.7)\end{array}$ & $\begin{array}{c}253 \\
(30.2)\end{array}$ & $\begin{array}{c}51 \\
(6.1)\end{array}$ & $\begin{array}{c}837 \\
(100.0)\end{array}$ \\
\hline $\begin{array}{l}\text { To attain prestige and } \\
\text { social status }\end{array}$ & $\begin{array}{c}139 \\
(16.6)\end{array}$ & $\begin{array}{c}133 \\
(15.9)\end{array}$ & $\begin{array}{c}283 \\
(33.8)\end{array}$ & $\begin{array}{c}194 \\
(23.2)\end{array}$ & $\begin{array}{c}88 \\
(10.5)\end{array}$ & $\begin{array}{c}837 \\
(100.0)\end{array}$ \\
\hline Impress others & $\begin{array}{c}190 \\
(22.7)\end{array}$ & $\begin{array}{c}186 \\
(22.2)\end{array}$ & $\begin{array}{c}225 \\
(26.9)\end{array}$ & $\begin{array}{c}186 \\
(22.2)\end{array}$ & $\begin{array}{c}50 \\
(6.0)\end{array}$ & $\begin{array}{c}837 \\
(\mathbf{1 0 0 . 0 )}\end{array}$ \\
\hline $\begin{array}{l}\text { Inherent features and } \\
\text { attributes of FMCGs } \\
\text { products }\end{array}$ & $\begin{array}{c}109 \\
(13.0)\end{array}$ & $\begin{array}{c}116 \\
(13.9)\end{array}$ & $\begin{array}{c}344 \\
(41.1)\end{array}$ & $\begin{array}{c}171 \\
(20.4)\end{array}$ & $\begin{array}{c}97 \\
(11.6)\end{array}$ & $\begin{array}{c}837 \\
(\mathbf{1 0 0 . 0 )}\end{array}$ \\
\hline
\end{tabular}

Source: Primary data

xvii. Influence of Branding Decisions in the process of buying a product:

\begin{tabular}{|l|l|l|}
\hline Brand Decision maker & Respondents & Percent \\
\hline Father/Father in-law & 353 & 42.2 \\
\hline Mother/Mother in-law & 232 & 27.7 \\
\hline Husband & 68 & 8.1 \\
\hline Wife & 53 & 6.3 \\
\hline Children & 30 & 3.6 \\
\hline All the above & 101 & 12.1 \\
\hline & $\mathbf{8 3 7}$ & $\mathbf{1 0 0}$ \\
\hline
\end{tabular}


It is stated that head of family will have high influence in buying decision especially as FMCG products fall in nonfood category. xviii. Factors influencing brand decision while purchasing products at the store:

\begin{tabular}{|c|c|c|c|c|c|c|c|c|}
\hline Factors & SDA & $\mathbf{D}$ & $\mathbf{N}$ & A & SA & Total & $\begin{array}{l}\text { Total } \\
\text { score }\end{array}$ & $\begin{array}{l}\text { Mean } \\
\text { score }\end{array}$ \\
\hline Product Display & $\begin{array}{c}158 \\
(18.9)\end{array}$ & $\begin{array}{c}166 \\
(19.8)\end{array}$ & $\begin{array}{c}168 \\
(20.1)\end{array}$ & $\begin{array}{c}192 \\
(22.9)\end{array}$ & $\begin{array}{c}153 \\
(18.3)\end{array}$ & $\begin{array}{c}837 \\
(\mathbf{1 0 0 . 0})\end{array}$ & 2527 & 3.02 \\
\hline $\begin{array}{c}\text { Product Endorsed by } \\
\text { (celebrity/famous } \\
\text { personality) }\end{array}$ & $\begin{array}{c}145 \\
(17.3)\end{array}$ & $\begin{array}{c}238 \\
(28.4)\end{array}$ & $\begin{array}{c}279 \\
(33.3)\end{array}$ & $\begin{array}{c}99 \\
(11.8)\end{array}$ & $\begin{array}{c}76 \\
(9.1)\end{array}$ & $\begin{array}{c}837 \\
(\mathbf{1 0 0 . 0})\end{array}$ & 2234 & 2.67 \\
\hline Product Range & $\begin{array}{c}114 \\
(13.6)\end{array}$ & $\begin{array}{c}93 \\
(11.1)\end{array}$ & $\begin{array}{c}233 \\
(27.8)\end{array}$ & $\begin{array}{c}294 \\
(35.1)\end{array}$ & $\begin{array}{c}103 \\
(12.3)\end{array}$ & $\begin{array}{c}837 \\
(\mathbf{1 0 0 . 0})\end{array}$ & 2690 & 3.21 \\
\hline $\begin{array}{l}\text { Recommendation by } \\
\text { Shopkeeper }\end{array}$ & $\begin{array}{c}82 \\
(9.8)\end{array}$ & $\begin{array}{c}264 \\
(31.5)\end{array}$ & $\begin{array}{c}206 \\
(24.6)\end{array}$ & $\begin{array}{c}231 \\
(27.6)\end{array}$ & $\begin{array}{c}54 \\
(6.5)\end{array}$ & $\begin{array}{c}837 \\
(\mathbf{1 0 0 . 0})\end{array}$ & 2422 & 2.89 \\
\hline Advertisement & $\begin{array}{c}70 \\
(8.4)\end{array}$ & $\begin{array}{c}151 \\
(18.0)\end{array}$ & $\begin{array}{c}304 \\
(36.3)\end{array}$ & $\begin{array}{c}233 \\
(27.8)\end{array}$ & $\begin{array}{c}79 \\
(9.4)\end{array}$ & $\begin{array}{c}837 \\
(\mathbf{1 0 0 . 0})\end{array}$ & 2611 & 3.12 \\
\hline Views of other customers & $\begin{array}{c}63 \\
(7.5)\end{array}$ & $\begin{array}{c}207 \\
(24.7)\end{array}$ & $\begin{array}{c}239 \\
(28.6)\end{array}$ & $\begin{array}{c}216 \\
(25.8)\end{array}$ & $\begin{array}{c}112 \\
(13.4)\end{array}$ & $\begin{array}{c}837 \\
(\mathbf{1 0 0 . 0})\end{array}$ & 2618 & 3.13 \\
\hline Product Availability & $\begin{array}{c}86 \\
(10.3)\end{array}$ & $\begin{array}{c}110 \\
(13.1)\end{array}$ & $\begin{array}{c}135 \\
(16.1)\end{array}$ & $\begin{array}{c}380 \\
(45.4)\end{array}$ & $\begin{array}{c}126 \\
(15.1)\end{array}$ & $\begin{array}{c}837 \\
(\mathbf{1 0 0 . 0})\end{array}$ & 2861 & 3.42 \\
\hline Other's Recommendation & $\begin{array}{c}70 \\
(8.4)\end{array}$ & $\begin{array}{c}205 \\
(24.5)\end{array}$ & $\begin{array}{c}228 \\
(27.2)\end{array}$ & $\begin{array}{c}255 \\
(30.5)\end{array}$ & $\begin{array}{c}79 \\
(9.4)\end{array}$ & $\begin{array}{c}837 \\
(\mathbf{1 0 0 . 0})\end{array}$ & 2579 & 3.08 \\
\hline Scheme / Discount & $\begin{array}{c}102 \\
(12.2)\end{array}$ & $\begin{array}{c}147 \\
(17.6)\end{array}$ & $\begin{array}{c}229 \\
(27.4)\end{array}$ & $\begin{array}{c}268 \\
(32.0)\end{array}$ & $\begin{array}{c}91 \\
(10.9)\end{array}$ & $\begin{array}{c}837 \\
(\mathbf{1 0 0 . 0})\end{array}$ & 2610 & 3.12 \\
\hline
\end{tabular}

Note: DA-Disagree,SDA-Strongly Disagree, N-Neither This infers that most of consumers buying FMCG products agree nor disagree, gave priority to product availability.

A-Agree, SA-Strongly Agree.

Source: Primary Data xix. Consumer perception of price towards FMCG products:

\begin{tabular}{|l|l|l|l|l|l|l|}
\hline Product & $\begin{array}{l}\text { Quite } \\
\text { Expensive }\end{array}$ & $\begin{array}{l}\text { Somewhat } \\
\text { Costly }\end{array}$ & $\begin{array}{l}\text { Value } \\
\text { for } \\
\text { money }\end{array}$ & Cheap & $\begin{array}{l}\text { I con't } \\
\text { consider } \\
\text { cost }\end{array}$ & Total \\
\hline Toothpaste & 32 & 138 & 472 & 15 & 180 & $\mathbf{8 3 7}$ \\
& $(3.8)$ & $(16.5)$ & $(56.4)$ & $(1.8)$ & $(21.5)$ & $(\mathbf{1 0 0 . 0 )}$ \\
\hline
\end{tabular}


Buying Behaviour and Preferences of Consumers with Reference to Fmcg Goods in North Coastal Andhra Pradesh

\begin{tabular}{|l|l|l|l|l|l|l|}
\hline Bathing soap & $\begin{array}{l}101 \\
(12.1)\end{array}$ & $\begin{array}{l}172 \\
(20.5)\end{array}$ & $\begin{array}{l}364 \\
(43.5)\end{array}$ & $\begin{array}{l}29 \\
(3.5)\end{array}$ & $\begin{array}{l}171 \\
(20.4)\end{array}$ & $\begin{array}{l}\mathbf{8 3 7} \\
\mathbf{( 1 0 0 . 0})\end{array}$ \\
\hline Hair shampoo & 65 & 249 & 316 & 48 & 159 & $\mathbf{8 3 7}$ \\
& $(7.8)$ & $(29.7)$ & $(37.8)$ & $(5.7)$ & $(19.0)$ & $\mathbf{( 1 0 0 . 0 )}$ \\
\hline Hair oil & 44 & 163 & 427 & 48 & 155 & $\mathbf{8 3 7}$ \\
& $(5.3)$ & $(19.5)$ & $(51.0)$ & $(5.7)$ & $(18.5)$ & $\mathbf{( 1 0 0 . 0 )}$ \\
\hline Mosquito Repellent & 64 & 121 & 351 & 114 & 187 & $\mathbf{8 3 7}$ \\
& $(7.6)$ & $(14.5)$ & $(41.9)$ & $(13.6)$ & $(22.3)$ & $\mathbf{( 1 0 0 . 0 )}$ \\
\hline
\end{tabular}

Source: Primary Data

xx. Commonalities:

\begin{tabular}{|l|l|l|}
\hline & Initial & Extraction \\
\hline Changing financial position & 1.000 & 0.899 \\
\hline Emerging beauty consciousness & 1.000 & 0.732 \\
\hline To protect health & 1.000 & 0.824 \\
\hline To be fashionable & 1.000 & 0.676 \\
\hline To live healthy and hygienic life & 1.000 & 0.836 \\
\hline To withstand pollution & 1.000 & 0.717 \\
\hline To change along with changes in civilization & 1.000 & 0.741 \\
\hline Sales and promotional activities of firms & 1.000 & 0.698 \\
\hline Low-unit price of FMCGs products & 1.000 & 0.813 \\
\hline Impressed by advertisements & 1.000 & 0.783 \\
\hline To attain prestige and social status & 1.000 & 0.827 \\
\hline Impress others & 1.000 & 0.733 \\
\hline Inherent features and attributes of FMCGs products & 1.000 & 0.659 \\
\hline
\end{tabular}

Each factor it is seen that out of the 12 variables 5 variables have value above 0.80 (table-.5.4.1(b)). Therefore only 5 variables obtained have exceeded the minimum acceptable MSA level and thus these variables were used as a criteria which influence buying behavior towards select FMCG products and are statistically significant. Hence, changing financial position of the consumers leads to buy a particular FMCG product in the market place. It is also found that the other factors of consumers buying select FMCG products are to protect health and live healthy and hygienic life. On the other hand low-unit price of FMCG products and to attain prestige and social status, consumers prefer to buy particular FMCG products. 


\begin{tabular}{|c|c|c|c|c|c|}
\hline & \multicolumn{5}{|c|}{ Component } \\
\hline Changing financial position & 0.140 & 0.119 & 0.232 & 0.202 & 0.878 \\
\hline Emerging beauty consciousness & 0.614 & 0.212 & 0.181 & 0.338 & 0.403 \\
\hline To protect health & $0.840^{*}$ & 0.168 & 0.137 & 0.265 & 0.027 \\
\hline To be fashionable & 0.758 & 0.137 & 0.275 & 0.004 & 0.087 \\
\hline To live healthy and hygienic life & $0.811^{*}$ & 0.214 & 0.333 & 0.123 & 0.072 \\
\hline To withstand pollution & 0.546 & 0.045 & 0.614 & 0.081 & 0.183 \\
\hline To change along with changes in civilization & 0.467 & 0.167 & 0.677 & 0.035 & 0.187 \\
\hline Sales and promotional activities of firms & 0.197 & 0.127 & 0.090 & 0.780 & 0.160 \\
\hline Low-unit price of FMCGs products & 0.106 & 0.254 & 0.176 & $0.835 *$ & 0.101 \\
\hline Impressed by advertisements & 0.092 & 0.559 & 0.509 & 0.387 & 0.231 \\
\hline To attain prestige and social status & 0.154 & $0.880^{*}$ & 0.143 & 0.095 & 0.007 \\
\hline Impress others & 0.096 & 0.779 & 0.049 & 0.230 & 0.250 \\
\hline Inherent features and attributes of FMCGs products & 0.229 & 0.737 & 0.219 & 0.119 & 0.043 \\
\hline
\end{tabular}

It shows that in the first priority of the factors the consumers of the FMCG buy a particular product to protect their health and to live healthy and hygienic life. In the second priority factor the consumers of the FMCG buy a particular product to attain prestige and social status. In the next priority factor the consumers of the FMCG buy a particular product with low-unit price of FMCGs products.

\section{MAJOR FINDINGS}

- It has been revealed that majority of the respondents felt that price is an important factor which they consider while purchasing the product. Out of the total respondents most of the respondents perceived the price of FMCG products as value for money followed by somewhat costly, don't consider cost, quite expensive and cheap.

- It has been observed that gender, education, income, family size and nature of family have an association with the sources of media through which they have come to know about FMCG products.

- It was depicted that most of the respondents felt that promotional offers such as buy two get one free were preferred over the other offers like price discount, bundled offer with another product and free gift. It can be inferred that with the evolution of organised retail schemes such as buy one get one (BOGO) have become very popular.

- It was observed that most of the respondents agreed that they will not visit more than one store to collect information about FMCG products as these are low price products and much information need not be gathered before buying the product.

- It has been depicted that product availability, product range are the factors influencing brand decisions while purchasing at the store followed by discounts, views of other customers, products endorsed by celebrity/famous personalities and products recommended by the shop keeper.

- It has been depicted that most of the respondents gave importance to brand while buying brands of toothpaste, bathing soap, hair shampoo, hair oil and mosquito repellent. Brands have become popular even in rural areas because of the advent of small packs in the above mentioned categories of products and display of these products have increased with the evolution of organised retail formats.

\section{CONCLUSION}

The research concludes that consumer awareness about FMCG products has increased and they are able to take decisions about which brand to buy and what type of promotions are available in various categories of products. They make planned purchases of quality products which is branded from the nearest store.Consumers used to shift to other brands of FMCG products if not satisfied through television advertisements also. 
In recent times consumers based in North Coastal Andhra Pradesh are accustomed to buy more of FMCG products and trying out various brands, so an understanding of the consumer behaviour enables the marketers to take marketing decisions which are in tune with consumer needs.

\section{REFERENCES}

1. Boylaud, Olivier and Giuseppe Nicoletti (2001), "Regulatory Reform in Retail Distribution”, OECD Economic Studies (32): 254273.

2. Boylaud, Olivier and Giuseppe Nicoletti (2001), "Regulatory Reform in Retail Distribution”, OECD Economic Studies (32): 254 273.

3. Cotterill, Ronald W., William P. Putsis Jr. and Ravi Dhar (2000), "Assessing the Competitive Interaction between Private Labels and National Brands", Journal of Business (73): 109-137.

4. Dobson, Paul W., Roger Clarke, Stephen Davies and Michael Waterson (2001), "Buyer Power and its Impact on Competition in the Food Retail Distribution Sector of the European Union", Journal of Industry, Competition and Industry (1): 247-281.

5. Morton, Fiona Scott and Florian Zettelmeyer (2004), "The Strategic Positioning of Store Brands in Retailer-Manufacturer Negotiations", Review of Industrial Organization (24): 161-194.

6. Steiner, Robert L. (2004), "The Nature and Benefits of National Bran/Private Label Competition", Review of Industrial Organization (24): 105-127.

7. Technopak Perspective (2009), A quarterly report by CIITechnopak, Vol. 2., pp. 1-2

8. Tokatli, Nebahat and Yonca Boyaci Eldener (2002), "Globalization and the Changing Political Economy of Distribution Channels in Turkey", Environment and Planning A (34): 217-238.

9. Chandrasekhar B.V.N.G (2013), Consumer Buying Behaviour and Brand Loyalty in FMCG, Journal of Business Management, Volume 2 Issue 2 Pg no 50-67.

10. Chikweche, T. and Fletcher, R. (2010). Understanding factors Chikweche, T. and Fletcher, R. (2010). Understanding factors

11. Deliya.M (2012), Consumer Behaviour Towards The New Packaging Of FMCG Products, Journal Of Research In Commerce and Management Vol no 1, Issue no 11 Pg no 199-211.

12. Garga, P., Ghuman, K., and Dogra, B., -Rural Marketing of Selected Fast Moving Consumer Goods in Punjabl, Indian Journal of Marketing, Vol. 39, No. 5, May 2009, pp. 21-27.

13. Jan.R (2013), A Study Of Brand Consciousness and Loyalty (FMCG), International Journal Of Contemporary Practices, Vol no 5, Issue 11, Pg no 8-26.

14. Kumarvel K and Vikkraman P(2013), A Study on Consumer Behaviour towards FMCG products with special reference to Personal Care Products, Life Science Journal, Vol No.10 Pg No 148149

15. Mahalingam.S and Kumar.P (2012), A Study on Consumer Behavior Towards Selected Fast Moving Consumer Goods In Coimbatore City, Indian Journal Of Education and Information Management Vpl no 5 Issue no 11,Pg no 500-507.

16. Mahavir Sehrawat and Subash C.Kundu (2007), Buying behaviour of rural and urban consumers in India: The impact of packaging, International journal of consumer studies pg630- 638. Consumer behaviour and brand preference of Britannia biscuits-An empirical study with respect to Salem, Tamil Nadu. Indian J.Marketing, 36(8):17-21.

18. Miremadi A and Faghani E(2012) A Empirical Study of Consumer Buying Behaviour and its influence on Consumer Preference in Iranian FMCG market: A Case Study International Business management Vol no 5 Pg no 146-152

19. Nandgopal, R. and Chinnaiyan, P., (2003), Brand preference of soft drinks in rural Tamil Nadu.Indian Journal of Marketing, 33(1):14- 17

20. PrashandiMital(2013), The effect of Brand Equity on Consumer Buying Behaviour in term of FMCG in Iran, Interdisciplinary Journal of Contemporary Research in Business, Vol No 4 Pg no 945957 Publishers, New York.

22. Viswanathan, M. and Rosa, J.A. (2010). Understanding subsistence marketplaces: toward sustainable consumption and commerce for a better world. Journal of Business Research, 63, pp. 535-537.

23. Viswanathan, M., Sridharan, S. and Ritchie, R. (2010), Understanding consumption and Entrepreneurship in subsistence marketplaces. Journal of Business Research, 63, pp. 570-581
17. Maruthamuthu, K., Krishnakumar, K. and Vasan, M., (2006),

21. Tyagi.C.L and Kumar.A (2004), Consumer Behaviour, Atlantic

24. William D. PERREAULT and E. Jerome Mc CARTHY (2002), Basic Marketing, McGraw-Hill, New York, 2002, p.161.

25. Yuvarani R (2013) A Study on Rural Consumer Behaviour towards Selected Fast Moving Consumer Goods in Salem District. International Journal of Scientific Research Vol no 2 Issue no 2 Pg no 14-15.

\section{AUTHORS PROFILES}

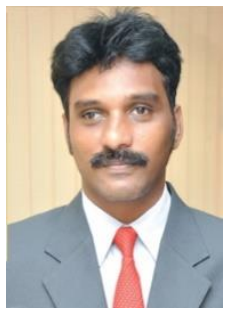

Dr. ShaikShamshuddin, with 18 years of teaching experience and few years of industry experience working as Assistant Professor, GITAM Institute of Management, GITAM Deemed to be University, Visakhapatnam. He is well expertise in Logistics and Supply Chain Management, Intellectual Property Rights, Marketing and Human Resource Management. $\mathrm{He}$ is also having consultancy and Research experience in various management areas. He worked as Assistant Coordinator for various Management Development and Executive Development programmes for HPCL, HSL, VPT, VSPTL, RPG, BHPV etc., He also published good number of Research papers in International reputed journals.

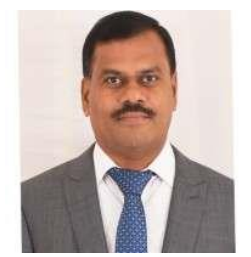

Dr. T. Venkateswarulu, A competent professional with 21years experience in industry and teaching in Retail Management, Strategic Management, Marketing/ Business Development, Network/ Franchisee Development as well as Key Account Management in Petroleum / FMCG sector. A proactive planner with expertise in market plan execution, market competitor and key account management \& financial planning relating to topline and bottom line as well as in Customer Relationship management. Proven ability of identifying and sourcing major institutional clients and escalating sales turnover. Experience in FMCG, Petroleum and Retail industries, handling various sales channels - Retail Sales, Institutional Sales and Operations. Excellent communication \& presentation skills with demonstrated abilities in training, team building \& driving front liners to highest echelons of performance.

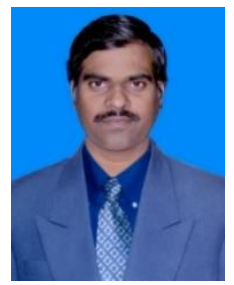

Dr U V Adinarayana Rao, is an Engineering and Management Post Graduate, having around 23 years of teaching experience including four years of international teaching exposure. He has published 20 articles in national and international peer reviewed journals and also two text book. He has attended good number of national and international conferences. His areas of interest include Research Methodology, Operations Management, and Marketing Management. He is an expert in SPSS Package. He is guiding five Ph. D. fellows and one thesis was awarded and two were submitted under his guidance. He is also Consultant to NTPC Need Assessment and Social Impact Assessments under CSR CD programmes across India. Upto now he has completed six consultancy projects.

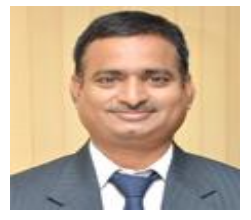

Dr. I Bangar Raju has a Doctorate in Commerce and Management,started corporate career in the year 1993 and served in different capacities up to the year 2000. Passion towards teaching made him to switch over profession from industry to teaching. He had 26 years ofservice both in industry (7 years) and academics (19 years). Corporate Finance, Strategy, Banking and Entrepreneurship subjects are of interest for teaching as well as for research. He is a resource person to SEBI for financial Literacy, NIMSME, DIC and MSME for entrepreneurial training. He extended training for more than 5000 prospective entrepreneurs. He is a consultant to NTPC for Need Assessment, Social Impact and Social perception studies on Corporate Social Responsibility. Currently he is working as Assistant professor in the Department of Entrepreneurship, GITAM Institute of Management, GITAM Deemed to be University, Visakhapatnam.

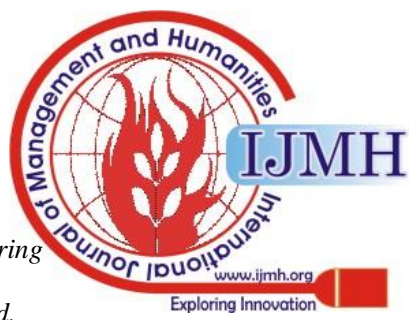




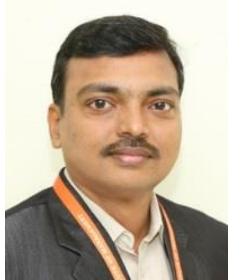

Dr. S. Gangadhara Rama Rao is Assistant Professor, GITAM Institute of Management, GITAM (Deemed to be University), Visakhapatnam. He obtained M.Com., MBA, M.Phil., and Ph.D. degrees from Andhra University, Visakhapatnam. $\mathrm{He}$ has participated in many Seminars/Workshops/FDPs organised by different Universities in in the country. He has 11 years of teaching experience in the areas of Finance and Marketing.

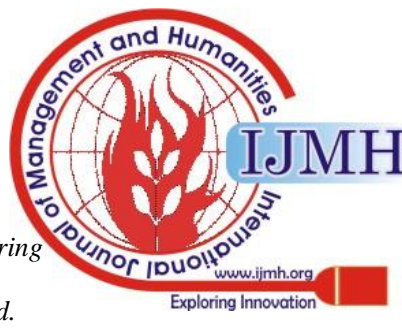

